\title{
METODOLOGIA PARA RASTREAMENTO COM MODIFICAÇÃO DOS ZEROS E REJEIÇÃO DE DISTÚRBIO APLICADA A SISTEMAS INCERTOS
}

\author{
Edvaldo Assunção* \\ edvaldo@dee.feis.unesp.br \\ Marcelo Carvalho Minhoto Teixeira* \\ marceloddee.feis.unesp.br
}

\author{
Cristiano Quevedo Andrea ${ }^{\dagger}$ \\ cristiano@batlab.ufms.br
}

João Onofre Pereira Pinto ${ }^{\dagger}$

jpinto@batlab.ufms.br

*UNESP - Universidade Estadual Paulista,

Departamento de Engenharia Elétrica,

Campus de Ilha Solteira,

15385-000, Ilha Solteira, São Paulo, Brasil.

${ }^{\dagger}$ UFMS - Universidade Federal de Mato Grosso do Sul,

Departamento de Engenharia Elétrica,

79074-460, Campo Grande, Mato Grosso do Sul, Brasil.

\begin{abstract}
The methodology for tracking system and disturbance rejection considering polytopic uncertainties in the plant is proposed in this work. The process of disturbance rejection is make though of the dynamic feedback of the system using a controller $K_{p}(s)$ that minimize the $\mathcal{H}_{2}$-norm guaranteed cost from $w(t)$ para $z(t)$, where $w(t)$ is the disturbance signal and $z(t)$ is the output of the system. And so, one use the optimal zeros modification with purpose of to design the tracking system. In this way, the modification of the zeros is used to minimize the $\mathcal{H}_{\infty}$-norm guaranteed cost from the reference input signal to the tracking error signal, where the tracking error signal was diference between the reference input signal and output signal of the system. The design is formulated in Linear Matrix Inequality (LMI) framework, when it presents a solution, the optimal solution of the stated control problem is obtained. Two practical examples illustrate the effectiveness of the proposed method.
\end{abstract}

\footnotetext{
Artigo submetido em 21/09/2005

1a. Revisão em 10/10/2006

2a. Revisão em 20/08/2007

Aceito sob recomendação do Editor Associado Prof. Liu Hsu
}

KEYWORDS: Zeros Modification, Control Systems, Tracking, Politopic Uncertainty, LMIs.

\section{RESUMO}

Propõe-se uma metodologia para rastreamento de sinais e rejeição a distúrbio aplicada a sistemas incertos, com incerteza do tipo politópica. Realiza-se o processo de atenuação do efeito do sinal de distúrbio na saída do sistema através da realimentação dinâmica da saída utilizando-se um controlador $K_{p}(s)$ que minimiza a norma $\mathcal{H}_{2}$ de custo garantido de $w(t)$ para $z(t)$, sendo $w(t)$ o sinal de distúrbio e $z(t)$ a saída do sistema. Então, para o rastreamento de sinais utiliza-se a modificação ótima dos zeros a fim de minimizar a norma $\mathcal{H}_{\infty}$ de custo garantido da função de transferência entre o sinal de referência e o sinal de erro de rastreamento, sendo o erro de rastreamento a diferença entre o sinal de referência $r(t)$ e o sinal de saída $z(t)$. O projeto de rastreamento de sinais e rejeição do efeito do ruído presente na planta é descrito na forma de LMIs que, quando factíveis, pode-se obter a solução ótima para o problema. Exemplos ilustram a viabilidade da metodologia proposta.

PALAVRAS-CHAVE: Modificação dos Zeros, Sistemas de 
Controle, Rastreamento, Incerteza Politópica, LMIs.

\section{INTRODUÇÃO}

Neste trabalho é proposta uma metodologia de projeto de sistema de controle baseada em Inequações Matriciais Lineares - LMIs (do inglês, Linear Matrix Inequalities) aplicada a sistemas incertos, com incerteza do tipo politópica. Neste contexto, projeta-se um sistema de rastreamento de sinais de referência com rejeição a distúrbio presente na planta. Realizase a atenuação do efeito do sinal de distúrbio na saída do sistema incerto através de um controlador $\mathcal{H}_{2}$ de custo garantido. Posteriormente, determina-se a posição ótima dos zeros com objetivo de minimizar a norma $\mathcal{H}_{\infty}$ de custo garantido do erro de rastreamento, sendo o sinal de erro de rastreamento a diferença entre o sinal de referência $r(t)$ e o sinal de saída $z(t)$.

$\mathrm{Na}$ teoria de sistema de controle, verifica-se que a alocação de pólos é amplamente utilizada em projetos de controle de realimentação de sistemas dinâmicos, vide por exemplo (Ogata, 1997) e (Chen, 1993). Contudo, observa-se um crescente uso dos zeros com a evolução das metodologias em sistemas de controle. Pode-se verificar, por exemplo, nos estudos científicos (Franklin et al., 1994), (Assunção et al., 2007b), (Assunção et al., 2007a) a utilização dos zeros aplicados em sistemas de controle. Ainda, observa-se a utilização dos zeros em redução de modelos (Hauksdóttir, 2000), projeto de controladores com características no domínio do tempo, tais como comportamento transitório (Moore and Bhattacharyya, 1990), robustez em sistemas de controle (Tu and Lin, 1992), entre outros temas.

Em sistemas de rastreamento, pode-se encontrar a aplicação da modificação dos zeros em (Assunção et al., 2004), onde é proposta uma metodologia de rastreamento de sinais e rejeição de ruídos, otimizando as normas $\mathcal{H}_{\infty}$ e $\mathcal{H}_{2}$ respectivamente. No entanto, em (Assunção et al., 2004), considera-se apenas sistemas determinísticos, isto é, não é considerada a presença de incertezas paramétricas da planta ao projeto de rastreamento de sinais.

Em (Mao and Yang, 1995) é apresentada uma metodologia para rastreamento de sinais para sistemas lineares incertos. A método de rastreamento apresentado em (Mao and Yang, 1995) propõe um sistema descentralizado de controle para o rastreamento robusto da saída para uma classe de sistemas incertos. A metodologia de rastreamento utilizada em (Mao and Yang, 1995) baseia-se no conceito de modelo interno, vide (Chen, 1998) para maiores detalhes. O rastreamento opera para uma determinada classe de sinais de referência e não existe nesta metodologia a rejeição de distúrbio presente na planta.
Recentemente, o uso de LMIs, como ferramenta, está sendo muito importante para a solução de um grande número de exemplos práticos em sistemas de controle, tais como, redução de modelo, projeto linear, não-linear, incertezas e sistemas com atraso (Boyd et al., 1994), (Assunção et al., 2007), (Assunção et al., 2007b), (Assunção and Peres, 1999), (Teixeira et al., 2005), (Teixeira et al., 2003), (Palhares et al., 2003), (Teixeira et al., 2006). As principais vantagens do uso das LMIs são que diferentes tipos de especificações de projeto e restrições podem ser descritas por LMIs, e o problema, quando existir solução, pode ser exatamente e eficientemente solucionado por algoritmos de otimização convexa (Boyd et al., 1994), (Nesterov and Nemirovsky, 1994). O ótimo global é encontrado através da convergência polinomial no tempo (El Ghaoui and Niculescu, 2000).

No processo de atenuação do efeito do sinal de distúrbio no sistema incerto utiliza-se uma metodologia de projeto de controlador já consagrada na literatura de sistemas de controle com otimização da norma $\mathcal{H}_{2}$ de custo garantido (Geromel et al., 1999). O método proposto para rastreamento de sinais utilizando modificação ótima dos zeros é formulado em termos de LMIs, o que resulta em uma descrição convexa do problema. O método considerado tem uma formulação mais simples que outras técnicas de rastreamentos para sistemas incertos encontradas na literatura, vide por exemplo, (Benson et al., 1992), (Shieh et al., 2003), (Koltchinski et al., 1998) e (Grasselli et al., 1992). O principal resultado é que o ótimo do problema é obtido, quando existir uma solução factível, com menor esforço computacional. Isto acontece, pois as LMIs podem ser facilmente resolvidas utilizando-se algoritmos de convergência polinomial disponíveis na literatura ((Gahinet et al., 1995), (Oliveira, 1999)). Exemplos ilustraram a viabilidade da metodologia proposta.

\section{FORMULAÇÃO DO PROBLEMA}

Considera-se o seguinte sistema linear e invariante no tempo com incertezas paramétricas descrito na forma de variáveis de estado:

$$
\begin{aligned}
\dot{x}(t) & =A(\alpha) x(t)+B_{2}(\alpha) u(t)+B_{1} w(t), \\
y(t) & =C_{2}(\alpha) x(t) \\
z(t) & =C_{1} x(t)
\end{aligned}
$$

sendo $A(\alpha) \in \Re^{n \times n}, B_{2}(\alpha) \in \Re^{n \times q}, B_{1} \in \Re^{n \times p}, C_{1} \in$ $\Re^{m \times n}, C_{2}(\alpha) \in \Re^{m \times n}, x(t)$ é o vetor de estados, $y(t)$ é a saída medida, $z(t)$ é a saída de referência, $u(t) \in \Re^{q}$ é a entrada de controle e $w(t) \in \Re^{p}$ e uma entrada exógena (do tipo distúrbio ou perturbação).

As matrizes dinâmicas incertas $A(\alpha), B_{2}(\alpha)$ e $C_{2}(\alpha)$ são representadas pela combinação convexa descrita a seguir 
(Boyd et al., 1994):

$$
A(\alpha)=\sum_{i=1}^{\kappa} \alpha_{i} A_{i}, B_{2}(\alpha)=\sum_{i=1}^{\kappa} \alpha_{i} B_{2 i}, C_{2}(\alpha)=\sum_{i=1}^{\kappa} \alpha_{i} C_{2 i}
$$

sendo $\alpha=\left[\alpha_{1} \cdots \alpha_{\kappa}\right]^{\prime}$ o vetor que parametriza o politopo de incertezas, e ainda,

$$
\sum_{i=1}^{\kappa} \alpha_{i}=1, \alpha_{i} \geq 0
$$

O número de vértices do politopo de incerteza do sistema varia de 1 até $\kappa$, sendo $\kappa=2^{\vartheta}$ e $\vartheta$ é o número de parâmetros incertos na planta.

\subsection{Problema 1}

O problema de rastreamento ótimo e rejeição de distúrbio utilizando a realimentação da saída considerando-se o sistema incerto descrito em (1), é minimizar a norma $\mathcal{H}_{2}$ de custo garantido entre a entrada exógena $w(t)$ e a saída $z(t)$. Neste processo deve-se projetar um controlador $K_{p}(s)$, que atenue o efeito do sinal de distúrbio na saída do sistema incerto. Para o rastreamento deve-se projetar parâmetros $M$ e $N$ de modificação ótima dos zeros que minimize a norma $\mathcal{H}_{\infty}$ de custo garantido entre a entrada de referência $r(t)$ e o erro de rastreamento $r(t)-z(t)$.

Observação 1 O diagrama de blocos do sistema de controle utilizado para resolver o Problema 1 é ilustrado na Figura 1, onde $K_{p}(s)=C_{p}\left(s I-A_{p}\right)^{-1} B_{p}$ é um compensador $\mathcal{H}_{2}$ para sistemas incertos utilizado na realimentação dinâmica da saída do sistema, $M$ é utilizado para resolver o problema da variação dos zeros, $N$ é uma matriz de ganho de rastreamento e $r(t)$ é o sinal de referência.

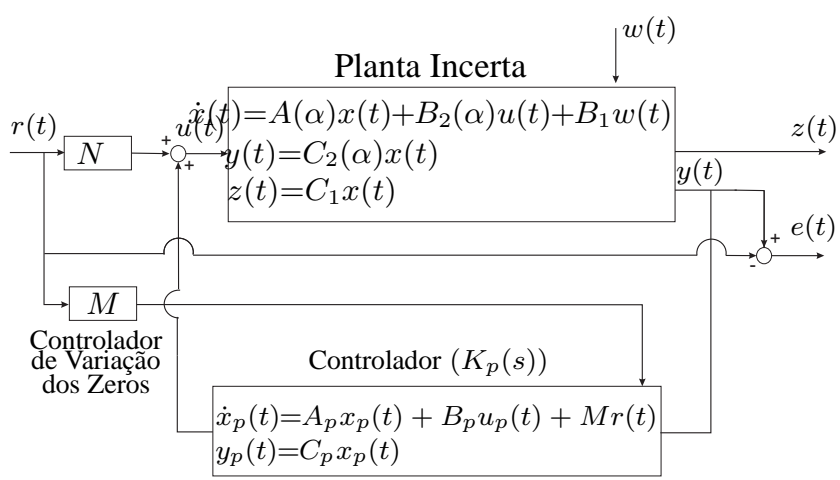

Figura 1: Controle ótimo de rastreamento e rejeição a distúrbio em sistemas incertos.

Pode-se atenuar o efeito do sinal de distúrbio ao sistema incerto através de um controlador $K_{p}(s)$ que realimenta a saída, conforme ilustrado na Figura 1. O projeto do controlador $K_{b}(s)$ é descrito em termos de LMIs, conforme o Teorema 1 proposto em Geromel et al. (1999).

Teorema 1 Considera-se o sistema incerto descrito em (1) com o controlador $\mathcal{H}_{2}$ de custo garantido utilizado na realimentação dinâmica da saída. Se existe solução para as LMIs descritas em (4), então pode-se obter a solução ótima para a norma $\mathcal{H}_{2}$ de $w(t)$ para $z(t)$ de custo garantido, minimizando-se o efeito do sinal de distúrbio no sistema, resolvendo-se

$$
\begin{aligned}
& \begin{array}{c}
\left\|H_{w z}\right\|_{2}^{2}=\min \\
\operatorname{s.a}\left[\begin{array}{ccc}
Y & I & B_{1} \\
I & X & X B_{1} \\
B_{1}^{\prime} & B_{1}^{\prime} X & W
\end{array}\right]>0,
\end{array} \\
& {\left[\begin{array}{ccr}
H_{i} & Z_{i}+M & Y C_{1}^{\prime} \\
Z_{i}^{\prime}+M^{\prime} & G_{i} & C_{1}^{\prime} \\
C_{1} Y & C_{1} & -I
\end{array}\right]>0} \\
& \delta>0 \text {, }
\end{aligned}
$$

sendo

$$
\begin{aligned}
H_{i} & =A_{i} Y+Y A_{i}^{\prime}+B_{2 i} L+L^{\prime} B_{2 i}^{\prime}, \\
G_{i} & =A_{i} X+X A_{i}+F C_{2 i}+C_{2 i}^{\prime} F^{\prime}, \\
Z_{i} & =A_{i}+Y A_{i}^{\prime} X+L^{\prime} B_{2 i}^{\prime} X+Y C_{2 i}^{\prime} F^{\prime},
\end{aligned}
$$

e ainda,

$$
L=C_{p} V^{\prime}, \quad F=U B_{p}, \quad e \quad M=V A_{p}^{\prime} U^{\prime} .
$$

As matrizes dinâmicas do controlador $\mathcal{H}_{2}, K_{p}(s)=C_{p}(s I-$ $\left.A_{p}\right)^{-1} B_{p}$, podem ser determinadas pela equação (6) com $U V^{\prime}=I-X Y$.

Prova: vide (Geromel et al., 1999).

Verifica-se no Teorema 1 , mais especificamente em (5), a existência de bilinearidades no projeto do compensador $\mathcal{H}_{2}$ de custo garantido para sistemas incertos. Este fator requer um elevado custo computacional para a solução deste problema. O algoritmo de decomposição cruzada (Tokunaga et al., 1996) tenta solucionar o problema para projeto de controladores $\mathcal{H}_{2}$ com custo garantido (Geromel et al., 1999). Inicialmente supõe-se que é conhecida uma solução factível inicial para o problema, e através das interações do algoritmo objetiva-se uma melhora em termos de custo da norma $\mathcal{H}_{2}$ para o projeto do controlador. Supõe-se que em uma iteração genérica $k$ as matrizes $Y_{k}, L_{k}, X_{k}$ e $F_{k}$ são soluções factíveis para o problema de otimização descrito na inequação (4). O teorema seguinte projeta o controlador $K_{p}(s)$ com custo garantido utilizando-se o algoritmo de decomposição cruzada para sistemas incertos em termos de LMIs (Geromel et al., 1999). 
Teorema 2 Considera-se o sistema (1) com o controlador $\mathcal{H}_{2}, K_{p}(s)=C_{p}\left(s I-A_{p}\right)^{-1} B_{p}$, de custo garantido utilizado para a realimentação dinâmica da saída. Se existe solução para as LMIs descritas em (7)-(8), então pode-se obter a solução ótima da norma $\mathcal{H}_{2}$ de $w(t)$ para $z(t)$ de custo garantido, minimizando-se o efeito do sinal de distúrbio no sistema, resolvendo-se

$$
\begin{aligned}
& \begin{array}{c}
\left\|H_{w z}\right\|_{2}^{2}=\min \\
\text { s.a } \quad\left[\begin{array}{ccc}
P & P & P B_{1} \\
P & X & X B_{1} \\
B_{1}^{\prime} P & B_{1}^{\prime} X & W
\end{array}\right]>0 \text {, }
\end{array} \\
& {\left[\begin{array}{ccc}
A_{k}^{\prime} P+P A_{k} & \Gamma & C_{k}^{\prime} \\
\Gamma^{\prime} & G & C_{1}^{\prime} \\
C_{k} & C_{1} & -I
\end{array}\right]<0,}
\end{aligned}
$$

sendo

$$
\begin{aligned}
\Gamma & =P A+A_{k}^{\prime} X+C_{2}^{\prime} F^{\prime}+S, \\
A_{k} & =A+B_{2} L_{k} Y_{k}^{-1}, \\
C_{k} & =C_{1} .
\end{aligned}
$$

Prova: vide (Geromel et al., 1999).

Por dualidade considera-se o problema de otimização convexa com variáveis $Y, L, Q, S$ e $W$ indicados nas equações (10) e (11):

$$
\begin{aligned}
& \begin{array}{c}
\left\|H_{w z}\right\|_{2}^{2}=\min \\
\text { s.a } \quad\left[\begin{array}{ccc}
Y & Q & B_{1} \\
Q & Q & B_{k} \\
B_{1}^{\prime} & B_{k}^{\prime} & W
\end{array}\right]>0,
\end{array} \\
& {\left[\begin{array}{ccc}
H & \Omega & Y C_{1}^{\prime} \\
\Omega^{\prime} & A_{k} Y+Y A_{k}^{\prime} & Q C_{1}^{\prime} \\
C_{1} Y & C_{1} Q & -I
\end{array}\right]<0}
\end{aligned}
$$

sendo

$$
\begin{aligned}
\Omega & =A Q+Y A_{k}^{\prime}+L^{\prime} B_{2}^{\prime}+S \\
A_{k} & =A+X_{k}^{-1} F_{k} C_{2}, \\
B_{k} & =B_{1} .
\end{aligned}
$$

É possível solucionar o problema do custo garantido para o controlador $\mathcal{H}_{2}$ através do algoritmo de decomposição cruzada, descrito a seguir:

Passo 1: Assume-se para uma iteração $k=0$ uma solução factível inicial com $Y_{0}, L_{0}, X_{0}, F_{0}, M_{0}$ e $W_{0}$ conhecidas. Para isto, considera-se que as matrizes $A_{i}, B_{2 i}$ e $C_{2 i}$ são conhecidas e pertencentes ao conjunto de incertezas politópicas, e então, a solução inicial é obtida resolvendo-se (4).

Passo 2: Com as matrizes $Y_{k}$ e $L_{k}$, resolve-se os problemas
(7) e (8). Determina-se o valor de $\alpha=\operatorname{tr}\left[W_{k}\right]$. A solução ótima fornece $F_{k}$ e $X_{k}$ a ser utilizada no passo 3 .

Passo 3: Com as matrizes $F_{k}$ e $X_{k}$, resolve-se os problemas (10) e (11). Determina-se o valor de $\beta=\operatorname{tr}\left[W_{k}\right]$. A solução ótima fornece $Y_{k}$ e $L_{k}$ a ser utilizada no passo 2 .

Passo 4: Se $\alpha_{k}-\beta_{k}<\epsilon, \epsilon>0$, finaliza-se o processo. Caso contrário, faça $k \rightarrow k+1$ e volte para o Passo 2 .

Com a resolução do processo iterativo obtém-se:

$$
L=L_{k}\left(P Y_{k}\right)^{-1}, \quad M=P^{-1} S, \quad F=\left(X_{k} Q\right)^{-1} F_{k},
$$

e, substituindo-se a equação (12) em (6) determina-se as matrizes dinâmicas do compensador $\mathcal{H}_{2}$ de custo garantido. Vide (Geromel et al., 1999) para maiores detalhes da convergência do algoritmo de decomposição cruzada.

O diagrama de blocos da Figura 1 pode ser descrito na forma de variáveis de estado $x(t)$ e $x_{p}(t)$, respectivamente estado da planta incerta e estado do controlador $\mathcal{H}_{2}$ de custo garantido, conforme a seguir:

$$
\begin{aligned}
{\left[\begin{array}{c}
\dot{x}(t) \\
\dot{x}_{p}(t)
\end{array}\right] } & =\left[\begin{array}{cc}
A(\alpha) & A_{u}(\alpha) \\
A_{l}(\alpha) & A_{p}
\end{array}\right]\left[\begin{array}{c}
x(t) \\
x_{p}(t)
\end{array}\right]+\left[\begin{array}{c}
B_{2}(\alpha) N \\
M
\end{array}\right] r(t)+\left[\begin{array}{c}
B_{1} \\
0
\end{array}\right] w(t), \\
z(t) & =\left[\begin{array}{ll}
C_{1} & 0
\end{array}\right]\left[\begin{array}{c}
x(t) \\
x_{p}(t)
\end{array}\right] \\
y(t) & =\left[\begin{array}{ll}
C_{2}(\alpha) & 0
\end{array}\right]\left[\begin{array}{c}
x(t) \\
x_{p}(t)
\end{array}\right] \\
e(t) & =r(t)-y(t)=r(t)-\left[\begin{array}{ll}
C_{2}(\alpha) & 0
\end{array}\right]\left[\begin{array}{c}
x(t) \\
x_{p}(t)
\end{array}\right],
\end{aligned}
$$

sendo

$$
\begin{aligned}
A_{u}(\alpha) & =B_{2}(\alpha) C_{p}, \\
A_{l}(\alpha) & =B_{p} C_{2}(\alpha) .
\end{aligned}
$$

Reescrevendo-se o sistema (13) de forma compacta, tem-se:

$$
\begin{aligned}
\dot{x}_{r}(t) & =A_{r} x_{r}(t)+B_{r} r(t)+B_{w i} w(t), \\
e(t) & =D_{r} r(t)-C_{y i} x_{r}(t), \\
y(t) & =C_{y i} x_{r}(t), \\
z(t) & =C_{r} x_{r}(t),
\end{aligned}
$$

sendo

$$
\begin{aligned}
x_{r}(t) & =\left[\begin{array}{c}
x(t) \\
x_{p}(t)
\end{array}\right], A_{r}=\left[\begin{array}{cc}
A(\alpha) & A_{u}(\alpha) \\
A_{l}(\alpha) & A_{p}
\end{array}\right], B_{r}=\left[\begin{array}{c}
B_{2}(\alpha) N \\
M
\end{array}\right], \\
B_{w i} & =\left[\begin{array}{c}
B_{1} \\
0
\end{array}\right], C_{r}=\left[\begin{array}{ll}
C_{1} & 0
\end{array}\right], C_{y i}=\left[\begin{array}{ll}
C_{2}(\alpha) & 0
\end{array}\right] \text { e } D_{r}=1 .
\end{aligned}
$$

Aplicando-se a transformada de Laplace no sistema (14), com condições iniciais nulas, obtém-se a função de transferência entre o sinal de entrada (entrada de referência e entrada exógena) e o sinal de saída do sistema conforme a seguinte equação:

$$
\begin{aligned}
& Z(s)=C_{r}\left(s I-A_{r}\right)^{-1} B_{r} R(s)+ \\
& C_{r}(s I-A)^{-1} B_{w i} W(s) .
\end{aligned}
$$


Para análise, considera-se um valor fixo para o parâmetro $\alpha$. De (16), a função de transferência $W(s) / Z(s)$ é dada por:

$$
\frac{W(s)}{Z(s)}=C_{r}\left(s I-A_{r}\right)^{-1} B_{w i}
$$

Verifica-se que norma $\mathcal{H}_{2}$ de $W(s)$ para $Z(s)$ pode ser minimizada devido ao projeto inicial do controlador $K_{p}(s)$, que é um compensador $\mathcal{H}_{2}$ de custo garantido para sistemas incertos; isto implica na minimização do efeito do sinal de distúrbio no desempenho do sistema.

Na Figura 1 observa-se a adição do termo $M r(t)$ na estrutura do compensador $\mathcal{H}_{2}$ de custo garantido para sistemas incertos. Os parâmetros $M$ e $N$ tem somente a função de alterar os zeros da função de transferência de $r(t)$ para $u(t)$ e não modifica os pólos estabelecidos pelo projeto inicial de $K_{p}(s)$, pois a função de transferência de $W(s)$ para $Z(s)$ não é modificada por $N$ ou $M$, vide equações (15) e (17). Com isso o desempenho do compensador $\mathcal{H}_{2}$ de custo garantido não é comprometido.

Para o projeto do rastreador ótimo, utiliza-se a transformada de Laplace no sistema (14), com condições iniciais nulas, obtém-se a função de transferência entre o sinais de entrada (entrada de referência e entrada exógena) e o sinal de erro, conforme a equação seguinte:

$$
\begin{array}{r}
E(s)=\left[-C_{r}\left(s I-A_{r}\right)^{-1} B_{r}+D_{r}\right] R(s)+ \\
C_{r}\left(s I-A_{r}\right)^{-1} B_{w i} W(s) .
\end{array}
$$

De (18), a função de transferência de $E(s) / R(s)$ é dada por:

$$
H_{m i}(s)=\frac{E(s)}{R(s)}=-C_{r}\left(s I-A_{r}\right)^{-1} B_{r}+D_{r} .
$$

Neste caso, através da modificação dos zeros pode-se projetar um rastreador de sinal minimizando a norma $\mathcal{H}_{\infty}$ de custo garantido da função de transferência entre o sinal de referência e o sinal de erro de rastreamento. O processo de modificação de zeros não interfere no projeto de rejeição de distúrbio, pois segundo (17) a função de transferência de $W(s)$ para $Z(s)$ não depende de $B_{r}$. Em (19) utiliza-se a posição dos zeros, implícitos na especificação de $N$ e $M$ em $B_{r}$, para o processo de minimização da norma $\mathcal{H}_{\infty}$ de custo garantido de (19).

\section{RASTREADOR DE SINAIS PARA SISTE- MAS INCERTOS}

Para o projeto do rastreador de sinais de controle para sistemas incertos, utilizou-se como critério de desempenho a norma $\mathcal{H}_{\infty}$. Ainda, o processo de otimização da norma $\mathcal{H}_{\infty}$ da função de transferência entre o sinal de referência e o sinal do erro de rastreamento $(r(t)-z(t))$ para sistemas incertos, com incertezas do tipo politópicas, é baseado no conceito de custo garantido, vide (Palhares et al., 1997) para maiores detalhes.

A norma $\mathcal{H}_{\infty}$ de um sistema contínuo, linear e invariante no tempo descrito pela função de transferência $H_{g}(s)=$ $C_{g}\left(s I-A_{g}\right)^{-1} B_{g}+D_{g}$ pode ser obtida pela solução, quando existir, do seguinte problema de otimização descrito na forma de LMIs (Boyd et al., 1994):

$$
\begin{aligned}
& \left\|H_{g}\right\|_{\infty}^{2}=\min \quad \delta \\
& \text { s.a } \quad\left[\begin{array}{ccc}
A_{g} Q+Q A_{g}^{\prime} & Q C_{g}^{\prime} & B_{g} \\
C_{g} Q & -I & D_{g}^{\prime} \\
B_{g}^{\prime} & D_{g} & -\delta I
\end{array}\right]<0, \\
& Q>0 \text {, } \\
& \delta>0 \text {. }
\end{aligned}
$$

A solução para o problema do rastreamento ótimo para sistemas incertos utilizando a variação dos zeros, consiste em determinar os parâmetros $M$ e $N$ que minimizam a norma $\mathcal{H}_{\infty}$ de custo garantido de $H_{m i}$ descrito em (19). Ainda inclui-se ao projeto o peso na freqüência, pois é interessante que o rastreador execute suas funções segundo uma faixa de freqüência especificada em projeto.

Para o projeto do rastreador de sinais para sistemas incertos com peso na freqüência (Valentin and Duc, 1997), deseja-se encontrar a solução global que otimize o problema descrito a seguir:

$$
\min \left\|H_{m i}(s) J(s)\right\|_{\infty},
$$

sendo $J(s)=\left(A_{j}, B_{j}, C_{j}\right)$ o peso na freqüência de saída, e considera-se $H_{m i}=\left(A_{r}, B_{r},-C_{r}, D_{r}\right)$ uma realização do sistema incerto linear invariante no tempo e estável indicado em (19). Na Figura 2 é ilustrada a estrutura de inclusão de peso na freqüência.

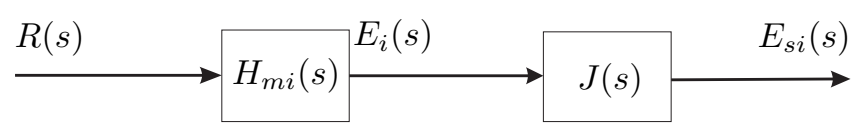

Figura 2: Projeto de sistemas de rastreamento aplicado a sistemas incertos com peso na frequiência.

Uma realização em espaço de estado de $E_{s i}(s) / R(s)=$ $H_{d i}=H_{m i}(s) J(s)$ para a estrutura ilustrada na Figura (2) pode ser expressa por:

$$
\left[\begin{array}{c|c}
A_{d i} & B_{d i} \\
\hline C_{d i} & D_{d i}
\end{array}\right]=\left[\begin{array}{cc|c}
A_{r} & 0 & B_{r} \\
-B_{j} C_{r} & A_{j} & B_{j} \\
\hline 0 & C_{j} & 0
\end{array}\right]
$$


Propõe-se o Teorema 3 para o projeto do rastreador com modificação dos zeros aplicados a sistemas com parâmetros incertos na planta.

Teorema 3 Considere o Problema 1 com a Observação 1. Se existe solução para as LMIs descritas em (23) então podese obter as matrizes $M\left(M \in \Re^{n \times p}\right)$ e $N\left(N \in \Re^{p \times p}\right)$ que minimizam a norma $\mathcal{H}_{\infty}$ de custo garantido do sistema $H_{m i}(s)=-C_{r}\left(s I-A_{r}\right)^{-1} B_{r}+D_{r}$ com peso na freqüência, resolvendo-se (23).

$O$ indíce $i$ em (23) denota o i-ésimo vértice do politopo de incertezas, $i=1,2, \cdots, \kappa ; \kappa=2^{\vartheta}$ e $\vartheta$ é o número de parâmetros incertos na planta.

Ainda, as matrizes $Q_{k l}=Q_{k l}^{\prime} ; k, l=1,2,3$, tem a mesma dimensão da matriz $A$, e para $k=3$ e $l=1,2,3$ as matrizes $Q_{k l}$ tem dimensões convenientes, e as matrizes $A_{j}, B_{j}$ e $C_{j}$ representam a dinâmica do filtro.

Prova: A inequação (23) é obtida considerando-se as matrizes $\left(A_{g}, B_{g}, C_{g}, D_{g}\right)=\left(A_{d i}, B_{d i}, C_{d i}, D_{d i}\right)$ na inequação (20), sendo a matriz $Q$ descrita da seguinte forma:

$$
Q=\left[\begin{array}{ccc}
Q_{11} & Q_{12} & Q_{13} \\
Q_{12}^{\prime} & Q_{22} & Q_{23} \\
Q_{13}^{\prime} & Q_{23}^{\prime} & Q_{33}
\end{array}\right]
$$

( $\left.A_{d i}, B_{d i}, C_{d i}, D_{d i}\right)$ dado por (22), sendo as matrizes $\left(A_{r}, B_{r}, C_{r}, D_{r}\right)$ mostradas em (19) e (15).

As matrizes $M$ e $N$ são soluções ótimas de (23) e minimizam a norma $\mathcal{H}_{\infty}$ de custo garantido da função de transferência entre o sinal de entrada de referência $r(t)$ e o sinal de erro de rastreamento $r(t)-z(t)$ para um sistema com parâmetros incertos na planta.

Os filtros utilizados no Teorema 3 são necessários somente em projeto, e posteriormente descartados para simulação ou implementação do sistema de rastreamento de sinais de referência.

\section{EXEMPLO 1}

Considera-se o sistema linear, invariante no tempo, com um parâmetro incerto na planta. O sistema apresenta uma incerteza do tipo politópica e a descrição na forma de espaço de estado é mostrada a seguir:

$$
\begin{aligned}
{\left[\begin{array}{c}
\dot{x}_{1}(t) \\
\dot{x}_{2}(t)
\end{array}\right] } & =\left[\begin{array}{cc}
-p & 0 \\
1 & 0
\end{array}\right]\left[\begin{array}{l}
x_{1}(t) \\
x_{2}(t)
\end{array}\right]+\left[\begin{array}{l}
1 \\
0
\end{array}\right] u(t)+\left[\begin{array}{l}
1 \\
0
\end{array}\right] w(t) \\
z(t) & =\left[\begin{array}{ll}
0 & 9
\end{array}\right]\left[\begin{array}{l}
x_{1}(t) \\
x_{2}(t)
\end{array}\right] \\
y(t) & =\left[\begin{array}{ll}
0 & 9
\end{array}\right]\left[\begin{array}{l}
x_{1}(t) \\
x_{2}(t)
\end{array}\right]
\end{aligned}
$$

sendo $x(t)$ o vetor de estados, $z(t)$ é a saída de referência, $y(t)$ é saída de medida, $u(t)$ o sinal de controle e $w(t)$ é o sinal de distúrbio adicionado ao sistema.

O parâmetro incerto $p$ descrito em (24) é uma incerteza politópica e apresenta-se da seguinte maneira:

$$
2<p<4
$$

Então, os politopos de incertezas são:

$$
A_{1}=\left[\begin{array}{cc}
-4 & 0 \\
1 & 0
\end{array}\right] \text { e } A_{2}=\left[\begin{array}{cc}
-2 & 0 \\
1 & 0
\end{array}\right]
$$

Deseja-se projetar um sistema de rastreamento de sinais com rejeição ao sinal de distúrbio para sinais de referências de baixa freqüência, até $10 \mathrm{rad} / \mathrm{seg}$, então é proposto o filtro $J_{1}(s)$ :

$$
J_{1}(s)=\frac{10}{0,01 s^{2}+01 s+1}
$$

Utiliza-se um compensador $\mathcal{H}_{2}$ de custo garantido, $K_{p}(s)$, para sistemas incertos com objetivo de atenuar o efeito do sinal de distúrbio na saída do sistema. Então, utiliza-se o Teorema 2 para a determinação do compensador $\mathcal{H}_{2}$, e o controlador $K_{p}(s)$ projetado é descrito a seguir:

$$
K_{p}(s)=-\frac{919603,08 s+706373210,17}{s^{2}+63,09 s+563657,69} .
$$

A norma $\mathcal{H}_{2}$ de custo garantido atingido em projeto é 0,027 . Este resultado implica na atenuação do efeito do sinal exógeno na saída do sistema.

Utiliza-se o Teorema 3 para o projeto dos parâmetro de modificação dos zeros $M$ e $N$ para sistemas incertos que proporcionam o rastreamento de sinais. Neste processo minimizase a norma $\mathcal{H}_{\infty}$ de $r(t)$ para $e(t)$ considerando-se sinais de baixa freqüência, até $10 \mathrm{rad} / \mathrm{seg}$, sendo $e(t)$ o sinal de erro entre a saída $z(t)$ e entrada de referência $r(t)$. A norma $\mathcal{H}_{\infty}$ obtida considerando-se todo o espectro de frequiência é 10,44. Analisando-se os resultados para a banda de freqüência especificada no problema, a maior magnitude de $\left|H_{m i}(j \omega)\right|$ é 0,0421 ; sendo $H_{m i}$ indicado em (19). Então o rastreador opera adequadamente para a banda de freqüência especificada em projeto.

A Figura 3 ilustra a resposta em freqüência de $E(s) / R(s)=$ $H_{m i}(s)$ para cada vértice do politopo de incertezas. Observase que a magnitude $E(s) / R(s)$ para a faixa de freqüência especificada em projeto é pequena, então, ocorre o processo de rastreamento nestas condições. Os parâmetros obtidos do 


$$
\left\|H_{d i}\right\|_{\infty}^{2}=\min \quad \delta
$$

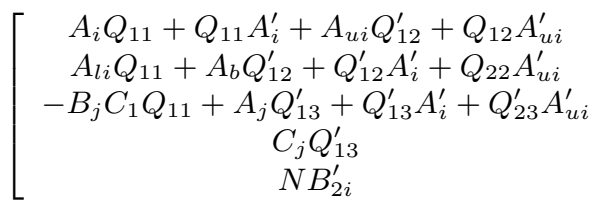$$
\begin{aligned}
& C_{j} Q_{13}^{\prime} \\
& N B_{2 i}^{\prime}
\end{aligned}
$$

$$
\begin{gathered}
A_{i} Q_{12}+A_{u i} Q_{22}+Q_{11} A_{l i}^{\prime}+Q_{12} A_{b}^{\prime} \\
A_{l i} Q_{12}+Q_{12}^{\prime} A_{l i}^{\prime}+A_{b} Q_{22}+Q_{22} A_{b}^{\prime} \\
-B_{j} C_{1} Q_{12}+A_{j} Q_{23}^{\prime}+Q_{13}^{\prime} A_{l i}^{\prime}+Q_{23}^{\prime} A_{b}^{\prime} \\
C_{j} Q_{23}^{\prime} \\
M^{\prime}
\end{gathered}
$$

$$
\begin{aligned}
& A_{i} Q_{13}+A_{u i} Q_{23}-Q_{11} C_{1}^{\prime} B_{j}^{\prime}+Q_{13} A_{j}^{\prime} \\
& A_{l i} Q_{13}+A_{b} Q_{23}-Q_{12}^{\prime} C_{1}^{\prime} B_{j}^{\prime}+Q_{23} A_{j}^{\prime} \\
& -B_{j} C_{1} Q_{13}-Q_{13}^{\prime} C_{1}^{\prime} B_{j}^{\prime}+Q_{33} A_{j}^{\prime}+A_{j} Q_{33} \\
& C_{j} Q_{33} \\
& {\left[\begin{array}{lll}
Q_{11} & Q_{12} & Q_{13} \\
Q_{12}^{\prime} & Q_{22} & Q_{23} \\
Q_{13}^{\prime} & Q_{23}^{\prime} & Q_{33}
\end{array}\right]>0,} \\
& \left.\begin{array}{cc}
Q_{13} C_{j}^{\prime} & B_{2 i} N \\
Q_{23} C_{j}^{\prime} & M \\
Q_{33} C_{j}^{\prime} & B_{j} \\
-I & 0 \\
0 & -\delta I
\end{array}\right]<0,
\end{aligned}
$$$$
\delta>0 \text {. }
$$

projeto de rastreamento para modificação dos zeros são:

$$
M=\left[\begin{array}{c}
183,35 \\
0,074
\end{array}\right], \text { e } N=1131,654
$$

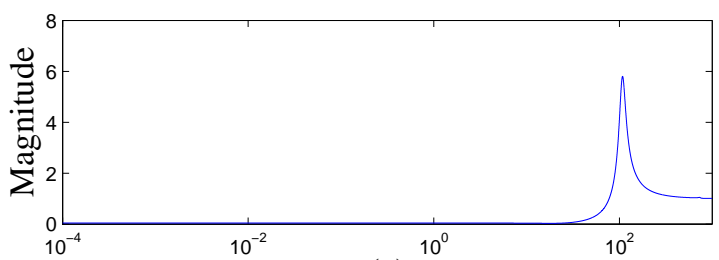

(a)

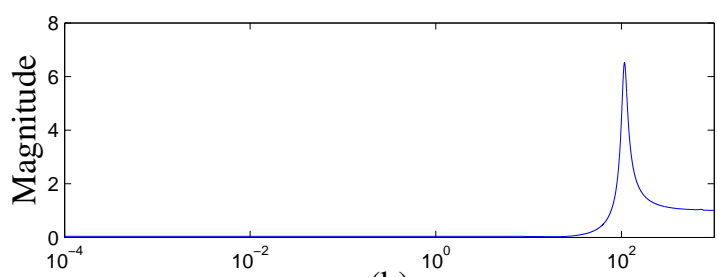

(b)

Freqüência $[\mathrm{rad} / \mathrm{seg}]$

Figura 3: Resposta em freqüência de $E(s) / R(s)=H_{m i}(s)$ : $\left|H_{m i}(j \omega)\right| \times \omega$. (a) primeiro vértice do politopo de incerteza, (b) segundo vértice do politopo.

Para a simulação do projeto do rastreador de sinais com rejeição de distúrbio considerou-se um sinal de referência $r(t)=\operatorname{sen}(10 t)$ e um sinal de perturbação do tipo distúrbio com amplitudes aleatórias não superiores a 1. A Figura
4 ilustra o resultado da simulação para o primeiro vértice do politopo de incerteza $\left(A_{1}\right)$ indicado em (25).

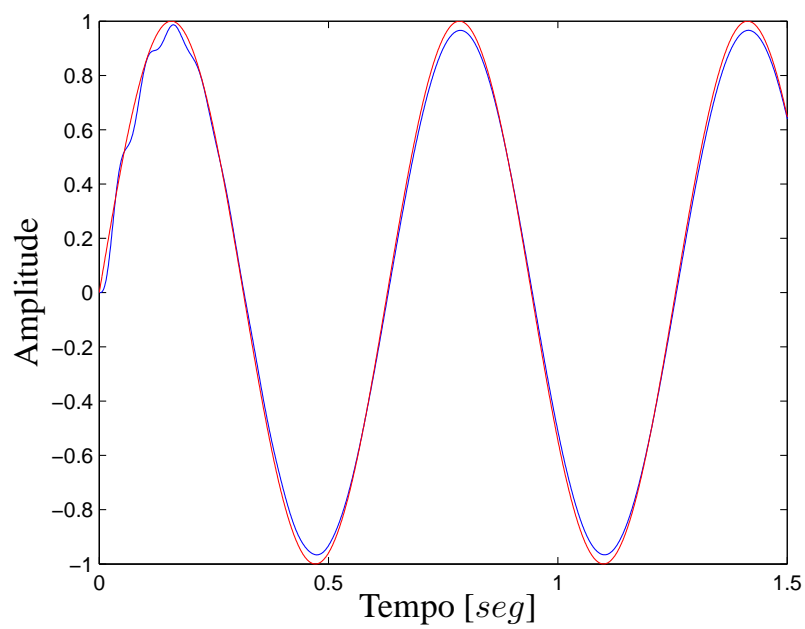

Figura 4: Sinal de saída $z(t)$ e sinal de entrada $r(t)$ estão quase sobrepostos.

Para este exemplo, os zeros do sistema foram: $-53,27 \pm$ $771,41 j$. Considerando o vértice do politopo de incerteza $\left(A_{1}\right)$, os pólos de malha fechada foram: $-9,03 \pm 106,98 j$; $-24,51 \pm 742,22 j$. Entretanto, considerando o vértice do politopo de incerteza $\left(A_{2}\right)$, os pólos de malha fechada do sistema foram: $-8,01 \pm 107,06 j ;-24,53 \pm 742,21 j$. 


\subsection{Exemplo 2}

Considere um sistema de um robô teledirigido (Sheridan, 1992) com incerteza politópica, e descrito na forma de espaço de estado da seguinte maneira:

$$
\begin{aligned}
{\left[\begin{array}{l}
\dot{x}_{1}(t) \\
\dot{x}_{2}(t) \\
\dot{x}_{3}(t)
\end{array}\right] } & =\left[\begin{array}{ccc}
-a & 0 & 0 \\
0 & -2 & 0 \\
0 & 0 & -3
\end{array}\right]\left[\begin{array}{l}
x_{1}(t) \\
x_{2}(t) \\
x_{3}(t)
\end{array}\right]+\left[\begin{array}{l}
1 \\
1 \\
0
\end{array}\right] u(t)+\left[\begin{array}{l}
1 \\
1 \\
0
\end{array}\right] w(t), \\
z(t) & =\left[\begin{array}{lll}
1 & 0 & 2
\end{array}\right]\left[\begin{array}{l}
x_{1}(t) \\
x_{2}(t) \\
x_{3}(t)
\end{array}\right] \\
y(t) & =\left[\begin{array}{lll}
1 & 0 & 2
\end{array}\right]\left[\begin{array}{l}
x_{1}(t) \\
x_{2}(t) \\
x_{3}(t)
\end{array}\right]
\end{aligned}
$$

sendo $x(t)$ o vetor de estados, $u(t)$ o sinal de controle e $w(t)$ o sinal de exógeno do tipo distúrbio ou ruído presente na planta.

O parâmetro incerto $a$ descrito em (26) é uma incerteza politópica e apresenta-se da seguinte maneira:

$$
-5<a<-0,5
$$

Então, os vértices do politopo de incertezas são:

$$
A_{1}=\left[\begin{array}{ccc}
-0,5 & 0 & 0 \\
0 & -2 & 0 \\
0 & 0 & -3
\end{array}\right] \text { e } A_{2}=\left[\begin{array}{ccc}
-5 & 0 & 0 \\
0 & -2 & 0 \\
0 & 0 & -3
\end{array}\right]
$$

Deseja-se projetar um sistema de rastreamento de sinais com rejeição ao sinal de distúrbio para sinais de referências de baixa frequiência, até $10 \mathrm{rad} / \mathrm{seg}$, então é proposto o filtro $J_{2}(s)$ :

$$
J_{2}(s)=\frac{168}{1 \times 10^{-6} s^{3}+0,001 s^{2}+0,01 s+1} .
$$

Utiliza-se um compensador $\mathcal{H}_{2}$ de custo garantido, $K_{p}(s)$, para sistemas incertos com objetivo de atenuar o efeito do sinal de distúrbio na saída do sistema. Então, utiliza-se o Teorema 2 para a determinação do compensador $\mathcal{H}_{2}$, e o controlador $K_{p}(s)$ projetado é descrito a seguir:

$K_{p}(s)=-\frac{4780762,292 s^{2}+74136629 s+261164543}{s^{3}+3800,876 s^{2}+76748,636 s+321550,881}$.

A norma $\mathcal{H}_{2}$ de custo garantido da função de transferência entre $w(t)$ e $z(t)$ para o sistema de malha fechada é 0,037 , implicando na atenuação do efeito do sinal de distúrbio na saída do sistema.
Objetivando-se o projeto do sistema de rastreamento, foi utilizada a metodologia proposta de modificação de zeros descrita no Teorema 3 , no qual a norma $\mathcal{H}_{\infty}$ de custo garantido da função de transferência de $r(t)$ para $e(t)$ é minimizada considerando-se sinais de faixa frequiência, até $10 \mathrm{rad} / \mathrm{s}$, para o sinal $r(t)$. A norma $\mathcal{H}_{\infty}$ de custo garantido obtida para todo o espectro de frequiência foi igual a 1,048, enquanto para a faixa de frequiência especificada em projeto, a maior magnitude de $\left|H_{m i}(j \omega)\right|$ foi 0,0688 , sendo $H_{m i}$ descrito em (19). Então o sistema de rastreamento opera adequadamente na faixa de freqüência especificada em projeto.

A Figura 5 ilustra a resposta em freqüência de $E(s) / R(s)=$ $H_{m i}(s)$ para os vértices do politopo de incertezas, e podese verificar para sinais de referência com freqüência até $10 \mathrm{rad} / \mathrm{s}$ que a norma $\mathcal{H}_{\infty}$ na faixa de frequiência especificada em projeto é adequada as características de um sistema de rastreamento. Os valores ótimos dos parâmetros $M$ e $N$ foram:

$$
M=\left[\begin{array}{c}
31,230 \\
-668,584 \\
53,821
\end{array}\right] \text { e } N=900,926 .
$$

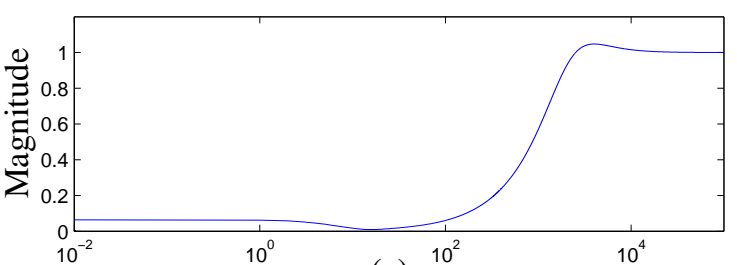

(a)

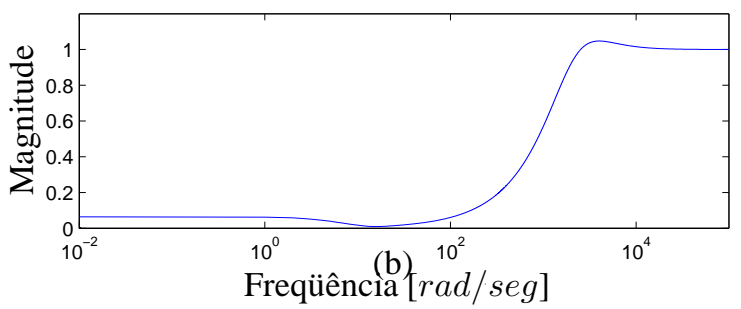

Figura 5: Resposta em freqüência de $E(s) / R(s)=H_{m i}(s)$ : $\left|H_{m i}(j \omega)\right| \times \omega$. (a) primeiro vértice do politopo $\left(A_{1}\right)$, (b) segundo vértice do politopo $\left(A_{2}\right)$.

Os vértices do politopo de incertezas foram descritos em (27). No processo de simulação foi considerado $r(t)=$ $\operatorname{sen}(2 t)$ como sinal de referência e um sinal de distúrbio $w(t)$ com amplitudes randômicas. Neste exemplo foi considerado que a máxima amplitude do sinal randômico era igual a 1 . O resultado de simulação para o primeiro vértice do politopo de incerteza $\left(A_{1}\right)$ é ilustrado na Figura 6.

Para este exemplo, os zeros do sistema foram: $-2 ;-1,991$; 


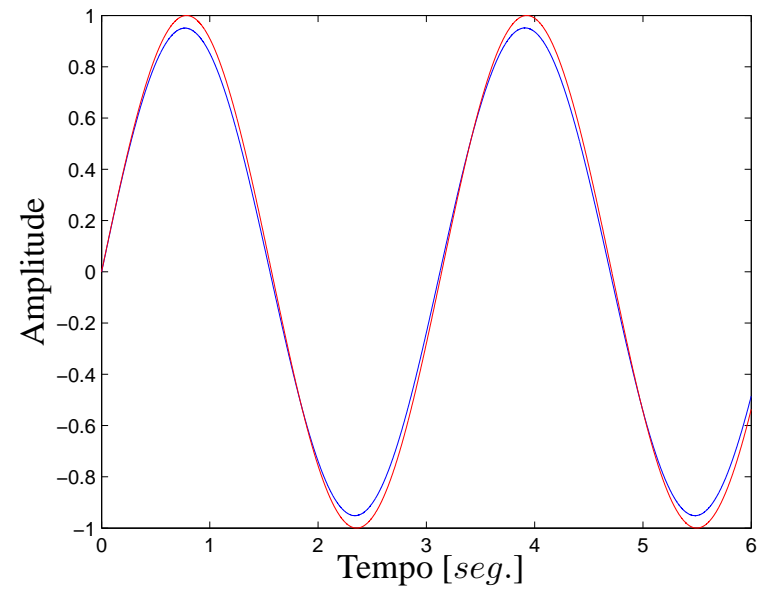

Figura 6: Sinal de saída $z(t)$ e sinal de entrada $r(t)$ quase sobrepostos.

-4,719; - 10,792 e -5332,391. Considerando o vértice do politopo de incerteza $\left(A_{1}\right)$, os pólos de malha fechada foram: $-2 ;-3 ;-5,406 ;-10,068 \mathrm{e}-1892,950 \pm 1103,407 j$. Entretanto, considerando o vértice do politopo de incerteza $\left(A_{2}\right)$, os pólos de malha fechada do sistema forma: $-2 ;-3$; $-5,410 ;-10,081$ e $-1895,192 \pm 1107,243 j$.

\section{CONCLUSÃO}

Neste trabalho foi proposto uma metodologia de rastreamento de sinais de referência e rejeição a distúrbio presente na planta incerta, com incerteza do tipo politópica. O processo de atenuação do efeito do sinal de distúrbio na saída do sistema foi realizado através de um controlador $\mathcal{H}_{2}$, sendo o projeto consagrado na literatura de sistemas de controle (Geromel et al., 1999), no qual minimizou-se a norma $\mathcal{H}_{2}$ de $w(t)$ para $z(t)$. Então, para o rastreamento de sinais utilizase a modificação ótima dos zeros a fim de minimizar a norma $\mathcal{H}_{\infty}$ da função de transferência entre o sinal de referência e o sinal de erro de rastreamento, sendo o erro de rastreamento a diferença entre o sinal de referência $r(t)$ e o sinal de saída do sistema $z(t)$.

A inclusão de peso na freqüência utilizado no projeto do rastreador possibilita ao projetista criar sistemas de rastreamento para sinais de referência em uma faixa de freqüência desejada. Desta modo, o sistema de rastreamento opera para faixas de freqüência determinada pelo projetista, como por exemplo rastreamento para sinais de baixa freqüência.

O projeto de rastreamento aplicado a sistemas incertos, com incerteza do tipo politópica, e rejeição a ruído presente na planta incerta são descritos na forma de LMIs, que quando factíveis, podem ser facilmente resolvidas através de algo- ritmos de convergência polinomial disponíveis na literatura ((Gahinet et al., 1995), (Oliveira et al., 1997)).

\section{AGRADECIMENTOS}

Os autores agradecem aos revisores pelos comentários úteis e construtivos e também à FAPESP e ao CNPq pelo apoio financeiro a esta pesquisa.

\section{REFERÊNCIAS}

Assunção, E., Andrea, C. Q. and Teixeira, M. C. M. (2004). Controle Ótimo $\mathcal{H}_{2}$ e $\mathcal{H}_{\infty}$ com Modificação de Zeros para o Problema de Rastreamento Usando LMI, Sba: Controle \& Automação Sociedade Brasileira de Automatica 15(4): 413-422.

Assunção, E., Marchesi, H. F., Teixeira, M. C. M. and Peres, P. L. D. (2007). Global Optimization for the $\mathcal{H}_{\infty}$ Norm Model Reduction Problem, International Journal of Systems Science 38(2): 125-138.

Assunção, E. and Peres, P. L. D. (1999). A Global Optimization Approach for the $\mathcal{H}_{2}$-norm Model Reduction Problem, Proceedings of the 38th IEEE Conference on Decision and Control, Phoenix, AZ, USA, pp. 18571862.

Assunção, E., Andrea, C. Q. and Teixeira, M. C. M. (2007a). Alocação de Zeros Aplicada a Sistema de Controle Via LMI, Sba: Controle \& Automação Sociedade Brasileira de Automatica . Aceito para publicação.

Assunção, E., Andrea, C. Q. and Teixeira, M. C. M. (2007b). $\mathcal{H}_{2}$ and $\mathcal{H}_{\infty}$-Optimal Control for the Tracking Problem with Zero Variation, IET Control Theory \& Applications 1(3): 682-688.

Benson, R. W., Schmitendorf, W. E. and Dolphus, R. M. (1992). Feedforward tracking for Uncertain Systems, Proceedings of the 31tf Conference on Decision and Control, Tucaon, Arizona, USA, pp. 2635-2639.

Boyd, S., El Ghaoui, L., Feron, E. and Balakrishnan, V. (1994). Linear Matrix Inequalities in Systems and Control Theory, SIAM Studies in Applied Mathematics, USA.

Chen, C. (1993). Analog \& Digital Control System Design, Sanders College Publishing, USA.

Chen, C. T. (1998). Linear System Theory and Design, Oxford University Press, USA.

El Ghaoui, L. and Niculescu, S. (2000). Advances in Linear Matrix Inequality Methods in Control, SIAM, Society for Industrial and Applied Mathematics, USA. 
Franklin, G. F., Powell, J. D. and Emami-Naeini, A. (1994). Feedback Control of Dynamic Systems, Addison Wesley, USA.

Gahinet, P., Nemirovski, A., Laub, A. J. and Chilali, M. (1995). LMI Control Toolbox User's Guide, The Mathworks Inc., USA.

Geromel, J. C., Bernussou, J. and Oliveira, M. C. d. (1999). $\mathcal{H}_{2}$-Norm Optimization with Constrained Dynamic Output Feedback Controllers: Decentralized and Reliable Control, IEEE Transactions on Automatic Control 44(7): 1449-1454.

Grasselli, O. M., Tornambe, A. and Longhi, S. (1992). Robust Tracking and Disturbance Rejection under Physical Parameter Uncertainties for Polynomialexponential Signals, Proceedings of the 31th Conference on Decision and Control, Tucson, Arizona, USA.

Hauksdóttir, A. S. (2000). Optimal Zeros for Model Reduction of Continuous-Time System, Proceedings of the American Control Conference, Chicago, Illinois, USA.

Koltchinski, V., Efromovick, V., Abdallah, C. T. and Heileman, G. L. (1998). Tracking Control of Uncertain Systems, Proceedings of the 37th IEEE Conference on Decision and Control, Tampa, Florida, USA.

Mao, C. L. and Yang, J. H. (1995). Decentralized Output Tracking for linear Uncertain Interconnected Systems, Automatica 31(1): 151-154.

Moore, K. L. and Bhattacharyya, S. P. (1990). A Technique for Choosing Zero Locations for Minimal Overshoot, IEEE Transactions on Automatic Control 35(5): 577580 .

Nesterov, Y. and Nemirovsky, A. (1994). Interior-Point Polynomial Algorithms in Convex Programming, SIAM Studies in Applied Mathematics, USA.

Ogata, K. (1997). Modern Control Engineering, 3th. edn, Prentice-Hall, New Jersey, USA.

Oliveira, M. C. (1999). Controle de Sistemas Lineares Baseados nas Desigualdades Matriciais Lineares, $\mathrm{PhD}$ Thesis, UNICAMP, Campinas, SP, Brazil.

Oliveira, M. C., Farias, D. P. and Geromel, J. C. (1997). LMISol, User's Guide, UNICAMP, Campinas-SP, Brasil. http://www.dt.fee.unicamp.br/.

Palhares, R. M., Hell, M. B., Durães, L. M., Ribeiro Neto, J. L., Teixeira, M. C. M. and Assunção, E. (2003). Robust $\mathcal{H}_{\infty}$ Filtering for a Class of State-delayed Nonlinear Systems in an LMI Setting, International Journal Of Computer Research 12(1): 115-22.
Palhares, R. M., Takahashi, R. H. C. and Peres, P. L. D. (1997). $\mathcal{H}_{\infty}$ and $\mathcal{H}_{2}$ Guaranteed Costs Computation for Uncertain Linear Systems., International Journal of Systems Science 28(2): 183-188.

Sheridan, T. B. (1992). Telerobotics, Automatica 25(4): 487507.

Shieh, N. C., Liang, K. Z. and Mao, C. J. (2003). Robust Output Tracking Control of an Uncertain Linear Systems via a Modified Optimal Linear-Quadratic Method, Journal of Optimization Theory and Applications 117(3): 649-659.

Teixeira, M. C. M., Assunção, E. and Palhares, R. M. (2005). Discussion on: $\mathcal{H}_{\infty}$ Output Feedback Control Design for Uncertain Fuzzy Systems with Multiple Time Scales: An LMI Approach, European Journal of Control 11(2): 167-169.

Teixeira, M. C. M., Assunção, E. and Avellar, R. G. (2003). On Relaxed LMI-Based Designs for Fuzzy Regulators and Fuzzy Observers, IEEE Transactions on Fuzzy Systems 11(5): 613-623.

Teixeira, M. C. M., Covacic, M. R. and Assunção, E. (2006). Design of SPR Systems with Dynamic Compensators and Output Variable Structure Control, 2006 IEEE International Workshop on Variable Structure Systems, Alghero - Italy, pp. 328-333.

Tokunaga, H., Iwasaky, T. and Hara, S. (1996). Multiobjective Robust Control with Transient Specifications, Proceedings of the 35th IEEE Conference Decision and Control, Kobe, Japan.

Tu, M. C. and Lin, C. M. (1992). Robust Stabilisation of Multivariable Feedback Systems with Desired Performance Requirement, IEE Control Theory and Applications 139(3): 259-266.

Valentin, C. and Duc, G. (1997). LMI-Based Algorithms for Frequency Weigth Optimal $\mathcal{H}_{2}$ - Norm Model Reduction, Proceedings of the 36th Conference on Decision and Control, San Diego, California, USA, pp. 767-772. 